\title{
Experiences of first PICC team in Slovenia
}

\section{J. Perme, MSc RN, E. Mihelj, RN, G. Pesek, Msc RN, Š. Unuk, RN University Medical Centre Ljubljana, Department of Infectious Diseases, Slovenia}

Introduction: The Department of Infectious Diseases of University Clinical Centre Ljubljana is 114 bed tertiary care facility. It provides diagnostic work up and treatment of serious infectious diseases in adults and children, including critically ill and severely immunocompromised patients. Difficult peripheral venous access is therefore a common problem at our department. More than 95\% of patients need parenteral antimicrobial treatment. Prolonged antibiotic treatment is expected in patients with infectious endocarditis, osteomyelitis, immunocompromised patients and foreign body related infections. In January 2017, four nurses from the intensive care unit attended workshops on ultrasound guided vascular access and PICC (peripherally inserted central catheter) insertion and acquired knowledge, which enabled them to establish PICC team. The action was supported by department management.

Aim: To evaluate the PICC team activity from February 2017 to February 2018. Evaluation includes number of performed procedures, fixation methods, catheter tip positioning after insertion and complications of the procedure.

Results: During this period 6959 patients were hospitalized and 245 PICC catheters were inserted. Sixty catheters (24\%) were inserted to ICU patients and 185 catheters (76\%) were placed in adult hospital wards patients. Eighty five percent of catheters were fixed to the skin with single stitch, $15 \%$ of catheters were suture free. Position of the catheters was verified with chest X-ray in all patients, correct position was achieved in $57 \%$ right after insertion. Peripheral vein thrombosis occurred in $0,8 \%$ of patients.

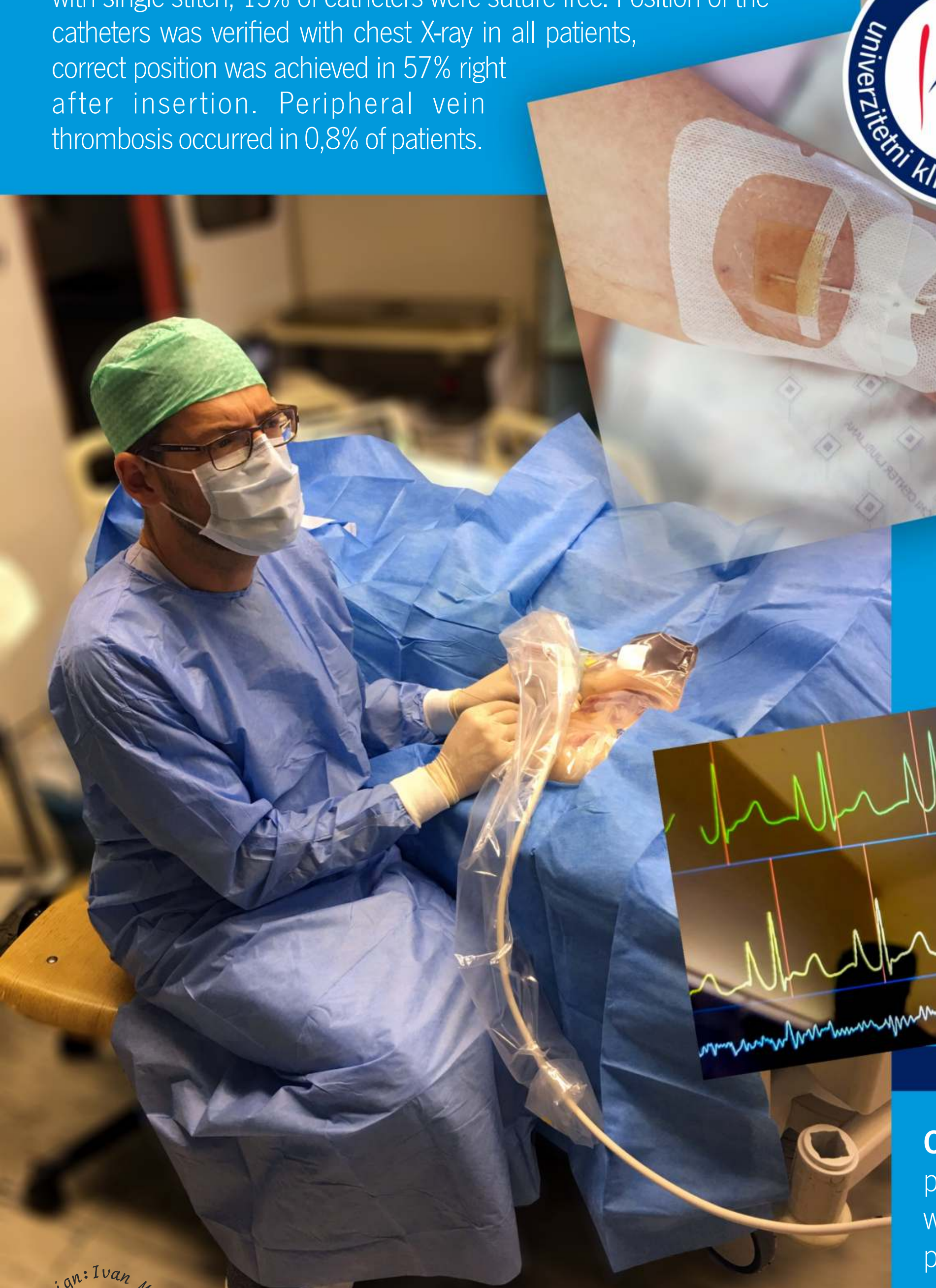

Conclusion: PICC team was the first team in Slovenia who started a project of PICC catheter insertion to hospitalized patients. During this period, we learned that central venous catheterization could be successfully performed by peripheral route. Due to low incidence of complications we plan to expand our experience to other hospitals in Slovenia. 